\title{
Pertussis Surveillance Trends in British Columbia, Canada, over a 20-year Period: 1993-2013
}

\author{
Chambers $\mathrm{C}^{1}$, Skowronski DM ${ }^{1 *}$, Hoang $\mathrm{L}^{2}$, Guiyun Li $\mathrm{H}^{1}$, Fritz $\mathrm{CE}^{1}$ Gustafson $\mathrm{R}^{3}$, \\ Murti $M^{4}$, Reid $A^{5}$, Parker $R^{6}$ and Bowering $D^{7}$
}

1 British Columbia Centre for Disease Control, Provincial Health Services Authority, Vancouver, BC

2 Public Health Microbiology Reference Laboratory, Provincial Health Services Authority, Vancouver, BC

3 Vancouver Coastal Health Authority, Vancouver, BC

$4 \quad$ Fraser Health Authority, Surrey, BC

$5 \quad$ Vancouver Island Health Authority, Victoria, BC

6 Interior Health Authority, Kelowna, BC

$7 \quad$ Northern Health Authority, Prince George, BC

* Corresponding author: danuta.skowronski@bccdc.ca

\section{Abstract}

Objective: To provide a surveillance update on overall and age-related pertussis trends in British Columbia (BC), Canada, spanning the 20-year period from 1993-2013.

Methods: Provincial surveillance data for confirmed pertussis cases were extracted from January 1, 1993 to October 31, 2013. Annual and age-specific incidence rates were derived using provincial and regional population estimates.

Results: BC experienced substantial pertussis epidemics in the late 1990s and early 2000s with incidence ranging from 20 to 40 per 100,000 overall and peaking in pre-teens aged 10-13 years at $>200$ per 100,000 during the epidemic of 2000. Overall incidence dropped to historical lows ranging from 1 to 6 per 100,000 between 2005 and 2011. This low-level activity was followed by resurgence in 2012 driven by outbreaks in Lower Mainland regions of $B C$ with overall provincial incidence reaching 10 per 100,000. Age-specific incidence in 2012 was highest among infants <1 year old (64 per 100,000) and children 12-13 years old (56-57 per 100,000), with a shift in the age distribution away from preschool-aged children toward pre-teens and young teens evident since 2000. Adult incidence remained $<10$ per 100,000 throughout the study period and was 5 per 100,000 in 2012. Year-todate provincial incidence rates overall for 2013 are 6 per 100,000, with ongoing asynchronous activity observed primarily on Vancouver Island.

Conclusions: Pertussis activity in BC showed expected cyclical fluctuations, with a peak incidence observed in 2012, mostly affecting infants and pre-teens/teens but at lower levels than prior peaks. Following substantial epidemics in the 1990s and early 2000s and the incorporation of acellular pertussis vaccine into the routine immunization program, the immuno-epidemiology of pertussis may still be in transition. Further monitoring and evaluation are needed to guide possible program changes for BC.

\section{Introduction}

Pertussis ("whooping cough") remains an endemic disease in Canada, as elsewhere, with cyclical peaks occurring every 2-5 years. ${ }^{1}$ Infants $<1$ year old are at higher risk for severe disease, including hospitalization, intensive care unit (ICU) admission and death, with the highest risk occurring in very young infants $<3$ months old. ${ }^{2 ; 3}$

Following the introduction of a routine paediatric immunization program using whole-cell pertussis formulation in Canada in 1943, pertussis incidence dropped by $>90 \%$, from an average of 165 cases per 100,000 in 1935-1939 to $\leq 10$ cases per 100,000 during the late 1980 s, and a historical low of 4 cases per 100,000 recorded in 1988 . ${ }^{4-6}$ However, despite universal paediatric immunization programs, pertussis incidence increased dramatically in Canada during the 1990 s and early 2000 s accompanied by a shift toward greater rates of infection in older 
children. ${ }^{4}$ In the westernmost province of British Columbia (BC), Canada, age-specific incidence during cyclical peaks in 2000 and 2003 were for the first time notably highest among pre-teens and young teens, with peak incidences of 150-300 cases per 100,000 in these age groups, and even surpassing rates reported among infants. ${ }^{7}$ This resurgent activity, and accompanying shift in the age profile, was largely attributed to advancing cohort effects stemming from the suboptimal effectiveness (20-60\%) of the adsorbed whole-cell vaccine product that was used in Canada from 1980 to $1997,{ }^{7-12}$ but also to waning immunity, increased awareness among clinicians, and improvements in diagnostic testing to include a more sensitive polymerase chain reaction (PCR) assay. ${ }^{4 ; 5 ; 7-9 ; 12}$

In response to these changing pertussis trends, most Canadian provinces and territories, including BC, switched to a more efficacious (>85\% efficacy after 3 doses) and less reactogenic 5-component acellular vaccine beginning in 1997. ${ }^{10 ; 13}$ Since then, acellular pertussis vaccine has been used for the routine paediatric immunization program in $\mathrm{BC}$ at 2, 4, 6 and 18 months of age and for the kindergarten booster dose at 4-6 years. A further adolescent booster dose in Grade 9 (14-15 years of age) was introduced in BC in 2004. (14 $^{4 ; 1}$

Following the substantial outbreaks of pertussis in the late 1990s and early 2000s, when provincial incidence peaked at 20-40 per 100,000 overall in BC, pertussis activity declined to lows of 1-6 per 100,000 between 2005 and 2011. ${ }^{3}$, This historically low activity in BC, however, was concurrent with widespread reporting of resurgent pertussis activity in the United States, ${ }^{15}$ notably in the corresponding west coast state of California in 2010 where overall incidence of $\sim 25$ cases per 100,000 was the highest reported in more than 50 years. ${ }^{16}$

In 2012 and 2013, BC experienced further cyclical peaks in pertussis activity affecting certain regions of the province. This surveillance update describes overall and age-related pertussis trends in BC spanning the 20-year period from 1993 to 2013. This perspective captures the population-level immuno-epidemiologic impact of previous epidemics and immunization program variations that may have potentially contributed to recent pertussis disease patterns

\section{Methods}

\section{Surveillance dataset}

Pertussis is a notifiable disease in BC. Cases of pertussis are reported to the BC Centre for Disease Control through the integrated Public Health Information System (iPHIS; all health authorities, with the exception of the Vancouver Coastal Health Authority (VCHA)) or the Primary Access Regional Information System (PARIS; VCHA only). Consistent with national notification requirements, this report describes epidemiologic trends based on confirmed case reporting only. Confirmed cases are those with a laboratory-confirmed (culture or PCR) infection with Bordetella pertussis or an epidemiological link to a laboratory-confirmed case with symptoms consistent with B. pertussis infection. ${ }^{17}$

PCR was introduced in April 1998 as a routine diagnostic test along with culture for pertussis by the BC Public Health Microbiology and Reference Laboratory (PHMRL), which provides the majority of pertussis testing in BC and contributes $\geq 85 \%$ of all confirmed cases detected in BC historically and during recent outbreaks. ${ }^{7}$ PCR has been accepted nationally alongside culture as evidence of laboratory confirmation since May 2000 . All laboratoryconfirmed cases of pertussis, regardless of testing laboratory, are notifiable to provincial public health and are reported in iPHIS/PARIS by health authorities.

Provincial surveillance data (age, sex, and geographic locators) were extracted from iPHIS/PARIS from January 1, 1993 to October 31, 2013 for all confirmed (laboratory-diagnosed or epidemiologically-linked) cases of pertussis reported in BC. Enhanced surveillance information pertaining to the 2012 outbreak was summarized locally within affected health authorities (Fraser Health Authority (FHA) and VCHA), including details such as immunization status and severe outcomes (e.g. hospitalizations and deaths). Cases were assigned to a regional health authority according to the local health unit that reported the case to provincial public health. Analysis of the monthly number of test requests to the PHMRL and pertussis test-positivity rates was based on data extracted from the Sunquest laboratory information system from January 1, 2011 to September 30, 2013. 
As data were collected for the purposes of public health surveillance, they are exempt from research ethics board approval.

\section{Statistical analysis}

Annual and age-specific incidence rates were calculated using provincial and regional population estimates from BC Stats (http://www.bcstats.gov.bc.ca/Home.aspx). Analyses were conducted using SAS version 9.3 (SAS Inc., Cary, NC).

\section{Results}

\section{Overall incidence trends, provincial}

Following peak epidemic periods in 1996, 2000 and 2003, pertussis incidence decreased to low-level endemic activity in $\mathrm{BC}$, with only minor periodic activity observed in limited geographic regions (e.g. Interior Health Authority (IHA) in 2006 and 2010 and Northern Health Authority and Vancouver Island Health Authority (VIHA) in 2008) (Figure 1). Overall provincial incidence reached historical lows of 3 per 100,000 in 2010 and one per 100,000 in 2011, followed by a cyclical resurgence in 2012, during which provincial incidence reached 10 per 100,000 overall. The 2012 peak incidence, however, remained substantially lower (less than half) compared to historical peaks observed in 1996 (23 per 100,000), 2000 (38 per 100,000) and 2003 (22 per 100,000) (Figure 1).

Figure 1. Annual incidence of confirmed pertussis cases in BC for the entire province and select health authorities, 1993 to $2013^{*}$

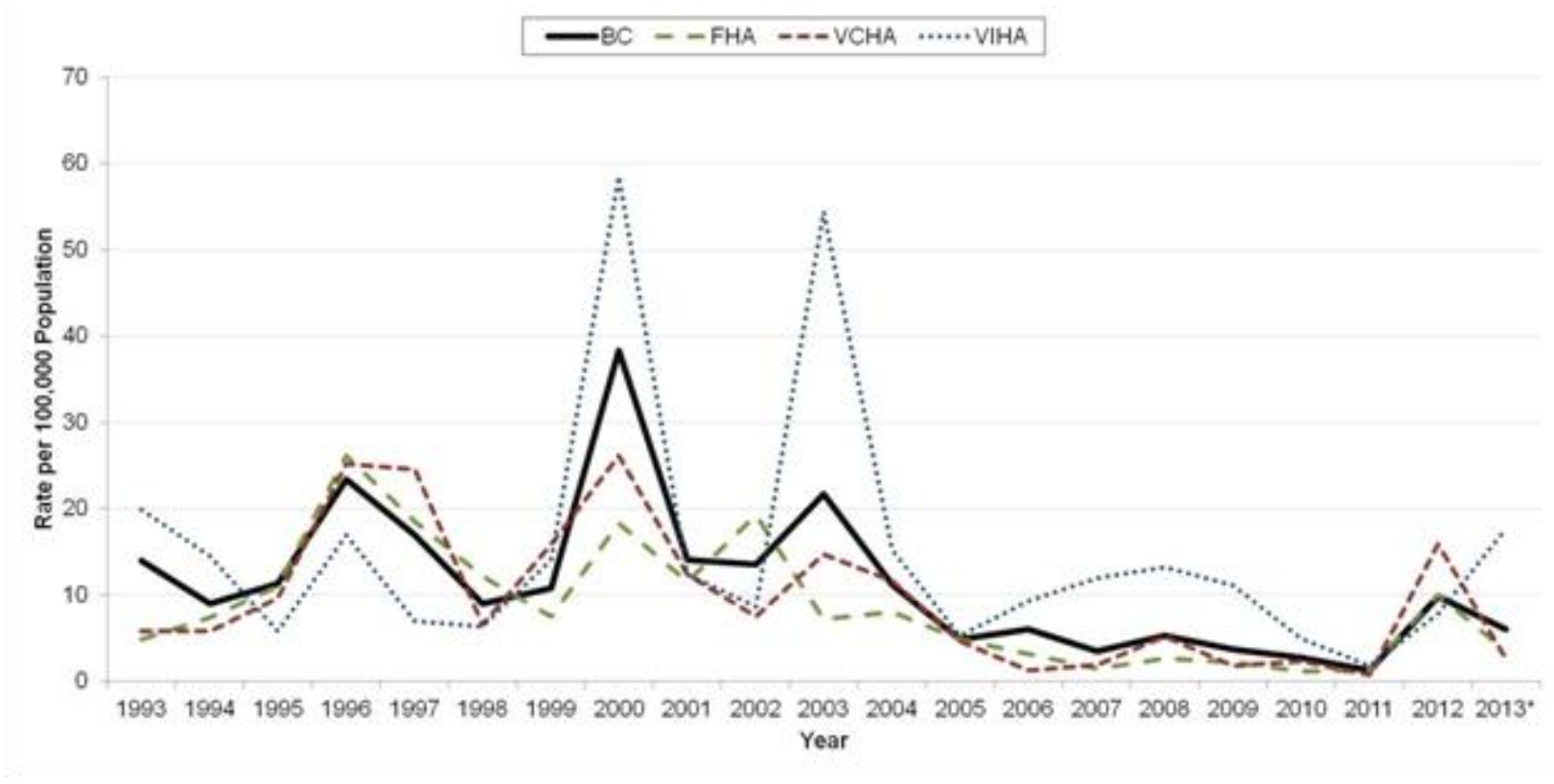

* Year-to-date (October 31, 2013); BC=British Columbia, FHA=Fraser Health Authority, VCHA=Vancouver Coastal Health Authority, VIHA=Vancouver Island Health Authority

Compared to 2011, the PHMRL received an almost 9-fold increase in pertussis test requests in 2012, averaging $>700$ tests per month in 2012 compared to 80 per month in 2011; on average, 320 tests were requested per month in 2013. However, the percent of tests positive for pertussis were comparable across all years and remained below $5 \%$ during heightened test volumes from February to April 2012, consistent with other causes of respiratory illness predominating during these winter months. Pertussis test-positivity rates peaked during July and August, concurrent with lower test volumes, in all three years (Figure 2). 
Figure 2. BC Public Health Microbiology and Reference Laboratory pertussis test requests and percent positive for pertussis by month and year, January 2011 to September 2013

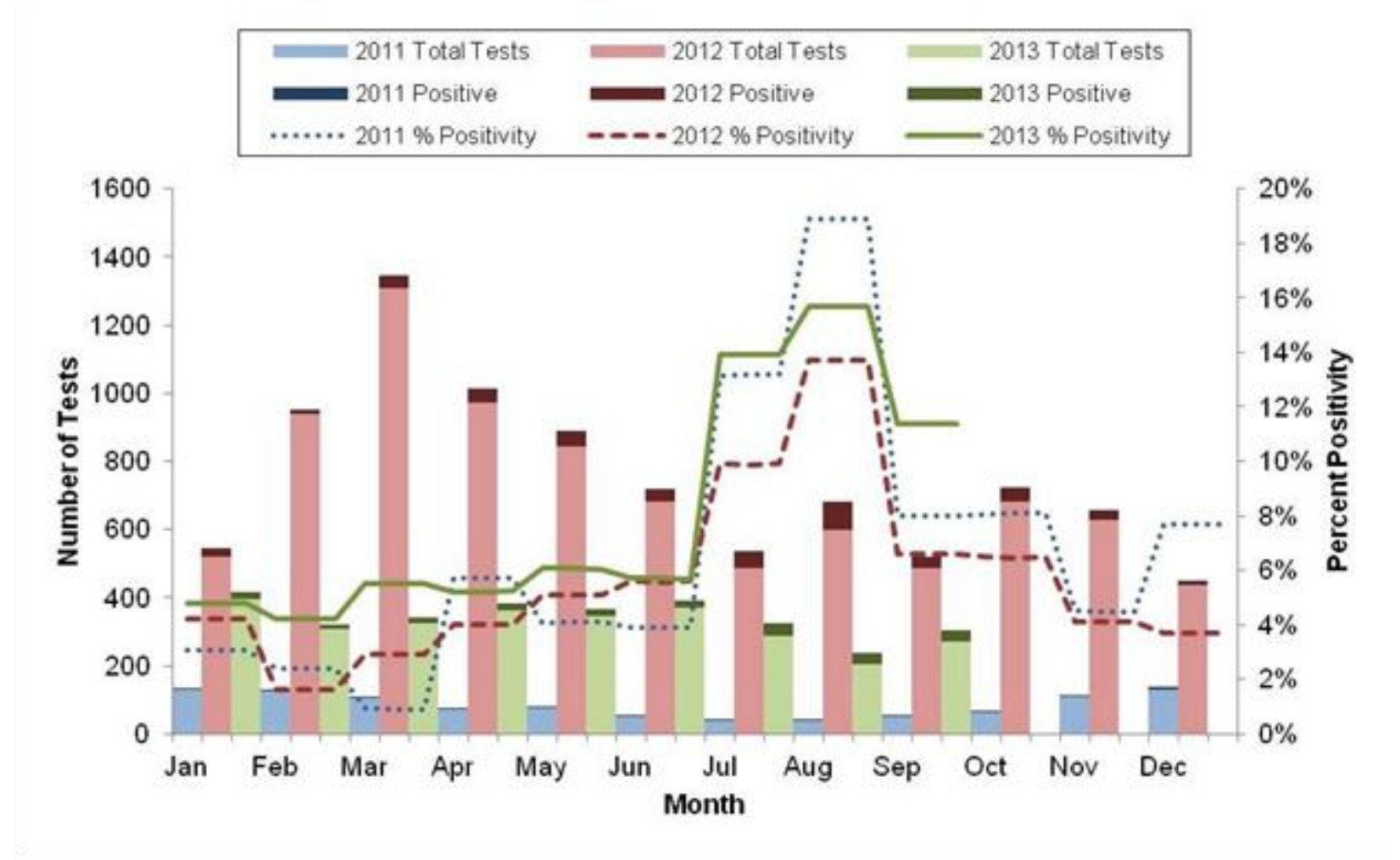

Age-specific incidence trends, provincial

Age-specific incidence rates in 2012 were highest among infants $<1$ year old (64 per 100,000) and children 12-13 years old (56-57 per 100,000) (Figure 3 ). Incidence rates were lowest in paediatric age groups immediately following the age of scheduled immunization booster doses: 16 per 100,000 in preschool-aged children 1-4 years and 12 per 100,000 in older teens aged 16-19 years (Figure 4A). Slightly higher incidence rates were observed in younger school-aged children 5-9 years at 28 per 100,000 (Figure 4A), with age-specific incidence gradually increasing with each additional year of age since the scheduled kindergarten booster dose (Figure 3). Similarly, incidence in teens aged 14-15 years around the timing of the scheduled Grade 9 booster dose was 28 per 100,000 (Figure 4A). Overall, incidence in 2012 was lowest among adults aged $\geq 20$ years at 5 per 100,000. 
Figure 3. Age-specific incidence of confirmed pertussis cases in BC for cyclical peak years, 1993, 1996, 2000, 2003, 2012 and 2013 (year-to-date)*

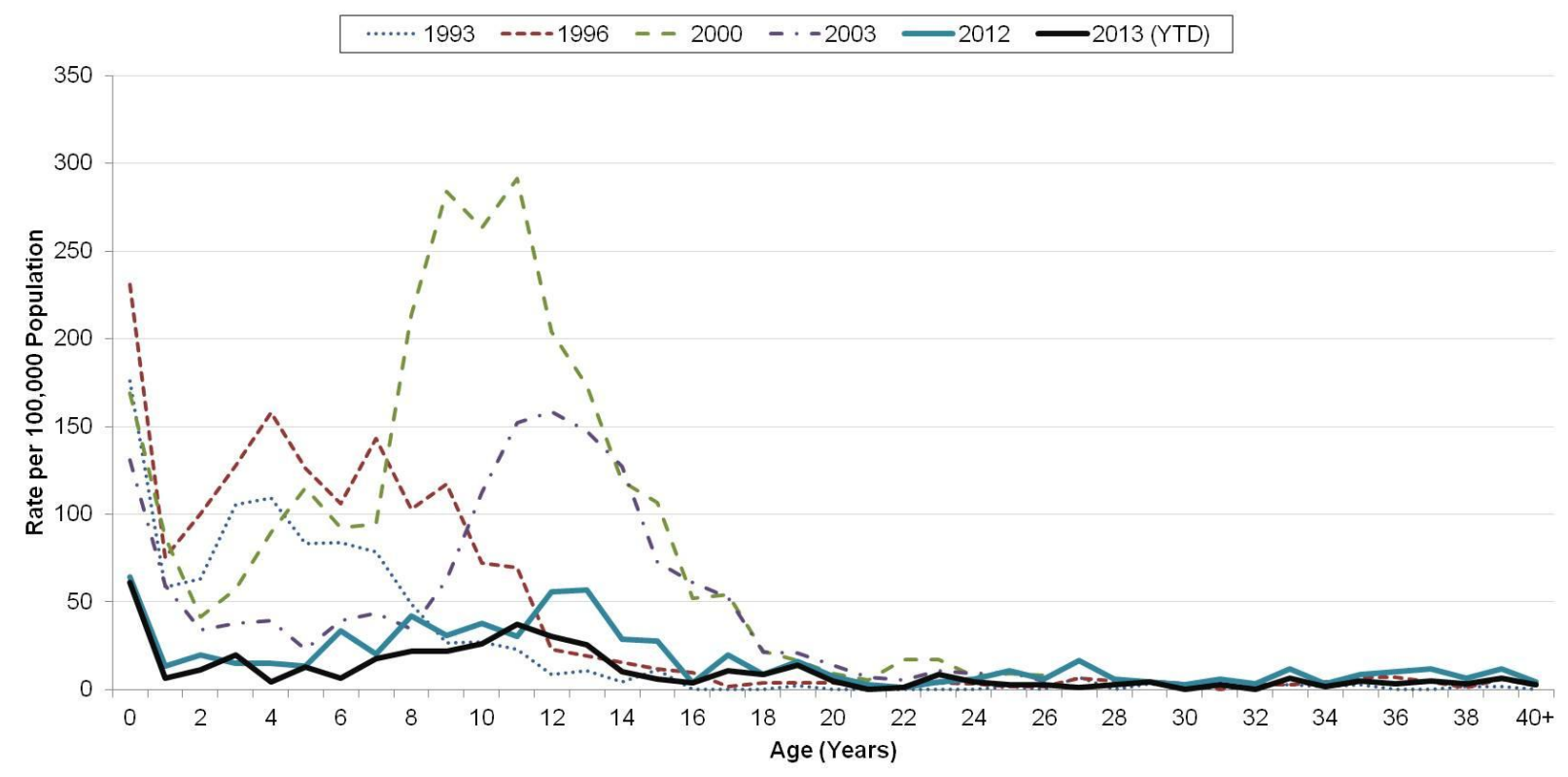

* Year-to-date (October 31, 2013) 
Figure 4. (A) Incidence rate and (B) proportion of confirmed pertussis cases in BC, by age group, 1993 to $2013^{*}$
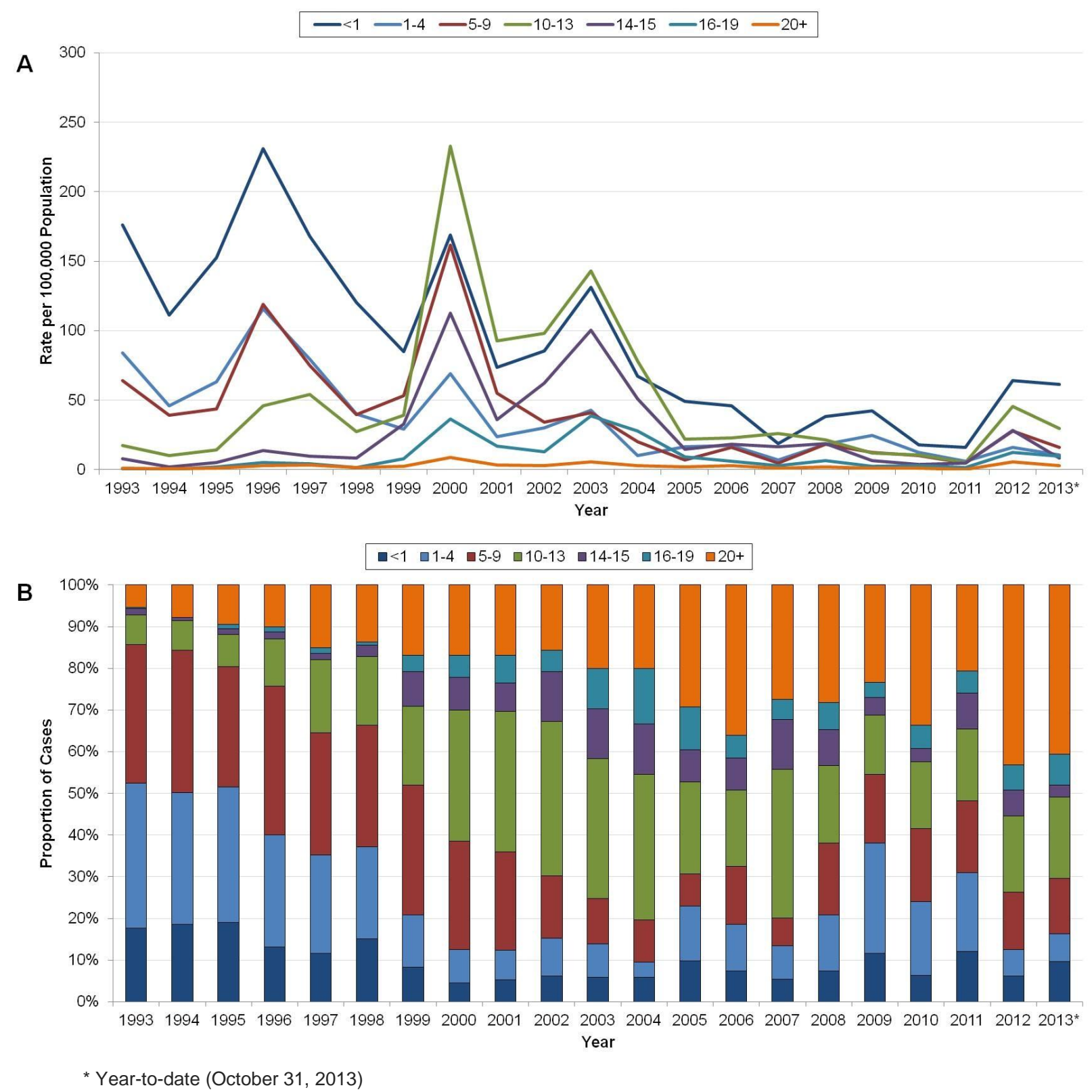

Of note, the higher incidence among pre-teens aged 10-13 years relative to infants $<1$ year old, first noted during the outbreak in $2000^{7}$, persisted in BC until 2005, after which rates were higher in infants. This difference is exemplified by incidence of 64 per 100,000 for infants and 46 per 100,000 for pre-teens observed during the 2012 peak (Figure 4A). The lower incidence among teens aged 16-19 years (12 per 100,000) and 14-15 years (28 per $100,000)$ relative to pre-teens aged $10-13$ years $(46$ per 100,000) in 2012 was already evident during prior nonepidemic and epidemic years of 1996 (5, 14, 46 per 100,000 respectively); 2000 (36, 113, 233 per 100,000 respectively); and 2003 (39, 100, 143 per 100,000 respectively), a pattern that notably preceded the introduction of the Grade 9 adolescent booster dose in 2004. 
In all paediatric age groups, incidence during the cyclical peak in 2012 was 3-6-fold lower compared to peak activity during the prior 20 -year period. Conversely, incidence in adults aged $\geq 20$ years has remained at more stable low levels, reported at 5 per 100,000 in 2012, less than a two-fold decrease compared to historical peak activity of 9 per 100,000 in 2000 . However, as a result of the dramatically declining incidence in all pediatric age groups, individuals aged $\geq 20$ years comprise a steadily increasing proportion of cases overall (Figure 4B).

\section{Regional outbreaks, 2012 and 2013}

The 2012 outbreak in BC was largely concentrated in the Lower Mainland region, with an overall incidence of 10 cases per 100,000 reported in FHA and 16 per 100,000 reported in VCHA, rates lower than prior cyclical peaks in these regions (Figure 1). Localized clusters of more intense activity contributed significantly to regional variation in rates. In the Fraser East area of FHA, pertussis incidence reached 24 per 100,000, and in VCHA, incidence in North Shore/Coast Garibaldi areas reached 36 per 100,000 (Figure 5).

Figure 5. Incidence of confirmed pertussis cases in BC, by Health Service Delivery Area (HSDA), 2012 and $2013^{*}$
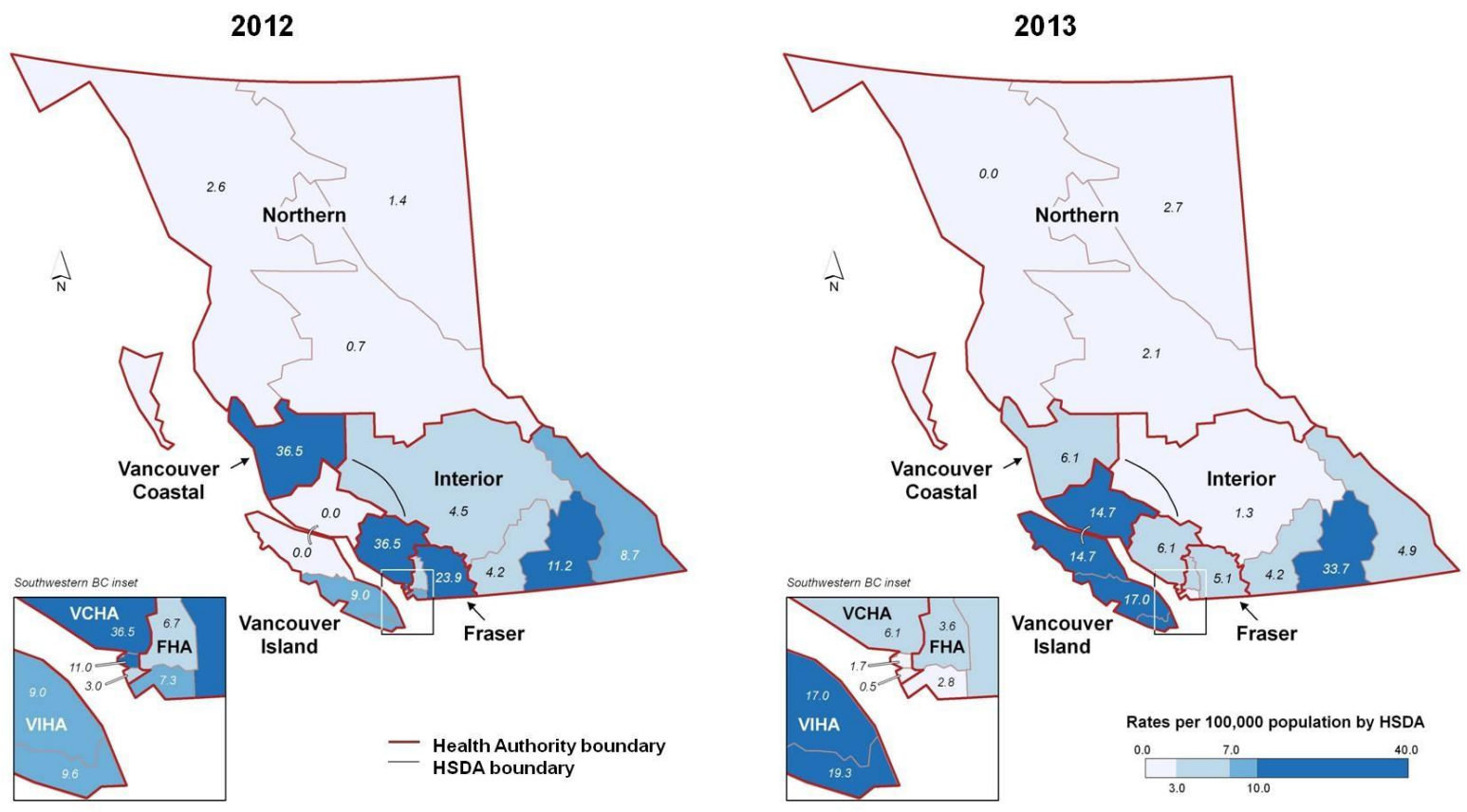

* Year-to-date (October 31, 2013)

More intense activity was associated with localized outbreaks at schools and other social gatherings, as well as pockets of low immunization coverage.

Since January 2013, incidence rates in FHA and VCHA have returned to expected endemic levels. Year-to-date incidence rates for 2013 are $\leq 5$ per 100,000 overall in each of these health authorities and are comparable to rates observed in prior non-epidemic years and overall in BC (Figure 1). In contrast, an asynchronous peak in pertussis activity was observed in VIHA in 2013. Year-to-date incidence rates in this region of 18 per 100,000 slightly exceed full-year rates observed during recent peak periods from 2007-2009 (ranging from 11-13 per 100,000), but are about three-fold lower than historical peaks observed in 2000 (59 per 100,000) and 2003 (54 per 100,000) (Figure 1). Activity in VIHA in 2013 has also been driven by local clusters of more substantial 
pertussis intensity. Localized outbreaks in the Kootenay Boundary area of IHA in 2013, with incidence reaching 34 per 1000,000 in this area, were also reported (Figure 5).

During the 2012 outbreaks in VCHA and FHA, less than half of paediatric cases aged 2 months to 20 years were up-to-date for immunization by age overall; a substantial proportion was either partially immunized or unimmunized ( $25 \%$ each) or had unknown immunization status $(4 \%)$. There were six hospitalizations among notified cases from these regions, including two patients who were admitted to ICU. All but one hospitalization occurred in infants $<3$ months old. An additional three hospitalizations were reported elsewhere in the province during 2012: two infants $<1$ year old and one infant $<3$ months old who was admitted to ICU. The confirmed pertussis infant hospitalization rate in 2012 both in outbreak regions and overall provincially in 2012 was 18 per 100,000 . There were no deaths notified in 2012; however, in 2013, a death in an immunized child $<3$ months old was reported. This is the first death due to pertussis to be identified in BC since 2005.

\section{Discussion}

This surveillance summary highlights trends in confirmed pertussis cases in BC over a 20 -year period spanning 1993-2013. Over this period, pertussis activity showed expected 2-5 year cyclical fluctuations. Confirmed pertussis notifications increased throughout the 1990s, reaching an apex in 2000, followed by a diminution in notifications across the subsequent decade. Following historical lows in 2010 and 2011, another cyclical, but less substantial, peak was observed in 2012. The shift in age distribution toward greater incidence in pre-teens than preschool-aged children, first highlighted during the 2000 outbreak in $\mathrm{BC},{ }^{7}$ has persisted through to the most recent period of peak activity.

In the pre-vaccine era, peak incidence was observed in pre-school-aged children and less than $20 \%$ of cases occurred in infants. ${ }^{7}$ In the post-vaccine era, peak incidence shifted downward to infants $<1$ year old, followed by a secondary peak in preschool-aged children, and accompanied by a decline in overall incidence. Since 2000, a further age-related shift is evident: infants $<1$ year old remain at high risk, but the secondary peak has shifted away from preschool-aged children toward pre-teens and young teens. During the most recent peaks in 2012 and 2013, this age-related pattern remains evident. However, the steep rise in incidence observed in pre-teens during the 2000 and 2003 outbreaks has been replaced by a more gradual relative incline from younger to older schoolaged children concurrent with lower-level activity overall.

Introduction of PCR diagnostic tests may have contributed to the dramatic increase in pertussis notifications during the late 1990s and into the early 2000 s in BC. PCR is considered more sensitive than culture-based diagnosis, extending the period of detection to six weeks or more following cough onset, although sensitivity is optimal within 2 weeks of cough onset. ${ }^{18-20}$ In particular, PCR may have increased detection among older children and adults who may have previously been missed by standard culture techniques due to delays in consultation for nonspecific, prolonged cough illness symptoms. The decline in pertussis notifications overall and across all age groups since 2000 , despite ongoing availability of PCR diagnosis, requires alternative explanation.

Epidemics in 2000 and 2003, accompanied by a shift in the age distribution toward older children, were previously explained by a cohort effect of accumulated initial susceptibility and subsequent waning immunity among prior recipients of the less-efficacious adsorbed whole-cell pertussis vaccine. ${ }^{5 ; 7 ; 1}$ In the 15 years since acellular vaccine was introduced into the routine pediatric immunization program, an increasing proportion of children comprise birth cohorts that received only acellular vaccine for all five of their prime-boost doses. In 2012 and 2013, individuals aged 14-15 years and younger would have been among the first cohorts of children to be fully immunized with only acellular vaccine. While pertussis overall remains well controlled in BC compared to historical activity, it is too early to assess the possible long-term impact of these changes to the routine pediatric immunization program on pertussis disease burden.

In addition to changes to the immunization program, low-level pertussis activity in BC may also reflect changes in population immuno-epidemiology resulting from the substantial provincial epidemics in 1996, 2000 and 2003. During the pre-vaccine era, almost all children would have been exposed to pertussis by their 12 th birthday. ${ }^{7}$ 
Whether acellular vaccine can sustain protection in the absence of widespread immune boosting from natural infection, which is thought to provide more durable protection than vaccine-induced immunity, remains to be seen. Recent observational studies conducted during outbreaks in the United States highlight rapid waning of protection from acellular vaccine within 2-5 years of the fifth booster dose. ${ }^{21-24}$ The age-related pattern of risk evident in our surveillance data, with gradually increasing incidence from 5 to13 years of age, is consistent with short-lived protection due to waning immunity. However, whether introduction of the adolescent booster to the provincial immunization program in 2004 attenuated the advancing cohort effect evident in pre-teens and teens cannot be resolved based on these data. Others have suggested that differences in contact networks and social mixing patterns by age may instead explain shifts in age-stratified incidence. ${ }^{25}$ The persistent pattern of reduced incidence in older teens, pre-dating recent immunization program changes, may support that assertion, although other factors, such as age-related differences in disease severity or health care-seeking behaviour, could also explain this finding. Given current pertussis activity levels in BC, and the increasing evidence of rapid waning of immunity following receipt of acellular vaccine booster doses, the value of additional doses and their optimal timing within the routine immunization schedule requires further evaluation.

\section{Limitation}

Limitations of pertussis surveillance in BC, as elsewhere, include reporting and misclassification biases. Only confirmed cases identified through routine passive surveillance were included in this summary; as such, true incidence is under-estimated. Because more sensitive PCR testing has been routinely implemented in BC since 1998, the incidence patterns observed over time since its implementation, notably a substantial decline in rates, likely reflect accurate trends, if not absolute risk. Within individual age-groups, decreasing incidence over time is also likely to be robust; however, comparisons across age-groups may be less reliable. Young infants present with more classical and severe illness and clinicians may be more likely to test, diagnose and/or report pertussis in very young infants. Atypical disease presentation in immunized children, older teens and adults may have contributed, in part, to their low observed incidence. Hospitalizations were based on confirmed pertussis cases specially notified as part of enhanced surveillance established during the outbreak in 2012. The hospitalization rate of 18 per 100,000 is lower than estimates based on clinical diagnosis extracted from administrative hospital discharge records during previous peaks in BC (ranging from 50 to 70 per 100,000). ${ }^{3}$ These data were not readily accessible for the current analysis. While the former may under-estimate, the latter is more likely to over-estimate pertussis hospitalization rates. Finally, asynchrony in the timing and geographic focus of cyclical peaks is not unexpected. Aggregation of surveillance data to health authority and provincial levels may obscure more intense or discrete activity at the local level.

\section{Conclusions}

Following substantial epidemics in 1996, 2000 and 2003, BC experienced historically low pertussis activity in 2010 and 2011, followed by regional outbreaks in 2012 that were concentrated in the Lower Mainland regions of the province. In 2013, pertussis activity has returned to endemic levels in these areas but with ongoing asynchronous activity localized within Vancouver Island and parts of the Interior. During cyclical peaks since 2000 , reported pertussis incidence has been highest in infants and pre-teens and lowest in adults. Recent epidemics and changes to the provincial immunization program may have altered age-related immunity patterns, while differences in social contact networks may affect exposure risks, together reflected in current surveillance trends. Despite recognized surveillance limitations, ongoing monitoring as well as active studies of age-related risk and vaccine effectiveness are needed and should be encouraged to inform possible changes to provincial programs and policies. 


\section{References}

1. Broutin H, Guegan JF, Elguero E, Simondon F, Cazelles B. Large-scale comparative analysis of pertussis population dynamics: periodicity, synchrony, and impact of vaccination. Am J Epidemiol. 2005;161(12):115967.

2. Bettinger JA, Halperin SA, De Serres G, Scheifele DW, Tam T. The effect of changing from whole-cell to acellular pertussis vaccine on the epidemiology of hospitalized children with pertussis in Canada. Pediatr Infect Dis J. 2007; 26(1):31-5.

3. Skowronski DM, Janjua NZ, Tsafack EP, Ouakki M, Hoang L, De Serres G. The number needed to vaccinate to prevent infant pertussis hospitalization and death through parent cocoon immunization. Clin Infect Dis. 2012; 54(3):318-27.

4. Public Health Agency of Canada. Part 4. Active vaccines. In: Canadian Immunization Guide. Ottawa: Public Health Agency of Canada; 2012.

5. Galanis E, King AS, Varughese P, Halperin SA. Changing epidemiology and emerging risk groups for pertussis. CMAJ. 2006; 174(4):451-2.

6. Tam TW, Bentsi-Enchill A. The return of the 100-day cough: resurgence of pertussis in the 1990s. CMAJ. 1998; 159(6):695-6.

7. Skowronski DM, De Serres G, MacDonald D, Wu W, Shaw C, Macnabb J, et al. The changing age and seasonal profile of pertussis in Canada. J Infect Dis. 2002; 185(10):1448-53.

8. Bentsi-Enchill AD, Halperin SA, Scott J, Maclsaac K, Duclos P. Estimates of the effectiveness of a whole-cell pertussis vaccine from an outbreak in an immunized population. Vaccine. 1997; 15(3):301-6.

9. De Serres G, Boulianne N, Duval B, Dery P, Rodriguez AM, Masse R, et al. Effectiveness of a whole cell pertussis vaccine in child-care centers and schools. Pediatr Infect Dis J. 1996; 15(6):519-24.

10. Gustafsson L, Hallander HO, Olin P, Reizenstein E, Storsaeter J. A controlled trial of a two-component acellular, a five-component acellular, and a whole-cell pertussis vaccine. N Engl J Med. 1996; 334(6):349-55.

11. Halperin SA, Bortolussi R, MacLean D, Chisholm N. Persistence of pertussis in an immunized population: results of the Nova Scotia Enhanced Pertussis Surveillance Program. J Pediatr. 1989; 115(5 Pt 1):686-93.

12. Ntezayabo B, De Serres G, Duval B. Pertussis resurgence in Canada largely caused by a cohort effect. Pediatr Infect Dis J. 2003; 22(1):22-7.

13. Zhang L, Prietsch SO, Axelsson I, Halperin SA. Acellular vaccines for preventing whooping cough in children. Cochrane Database Syst Rev. 2012; 3:CD001478.

14. National Advisory Committee on Immunization (NACl). Prevention of pertussis in adolescents and adults. Can Commun Dis Rep. 2003; 29(ACS-5):1-9.

15. Cherry JD. Epidemic pertussis in 2012--the resurgence of a vaccine-preventable disease. N Engl J Med. 2012; 367(9):785-7.

16. California Department of Public Health. Pertussis report. May 16, 2011 [Internet]. Sacramento: California Department of Public Health; 2011 [cited 18 Oct 2013]. http://www.cdph.ca.gov/programs/immunize/Pages/PertussisSummaryReports.aspx.

17. BC Centre for Disease Control. Management of specific diseases - pertussis. In: British Columbia Communicable Disease Manual. Vancouver: BC Centre for Disease Control, 2010. p. 1-36.

18. He Q, Schmidt-Schlapfer G, Just M, Matter HC, Nikkari S, Viljanen MK, et al. Impact of polymerase chain reaction on clinical pertussis research: Finnish and Swiss experiences. J Infect Dis. 1996;174(6):1288-95.

19. Sotir MJ, Cappozzo DL, Warshauer DM, Schmidt CE, Monson TA, Berg JL, et al. Evaluation of polymerase chain reaction and culture for diagnosis of pertussis in the control of a county-wide outbreak focused among adolescents and adults. Clin Infect Dis. 2007;44(9):1216-9. 
20. van der Zee A, Agterberg C, Peeters M, Mooi F, Schellekens J. A clinical validation of Bordetella pertussis and Bordetella parapertussis polymerase chain reaction: comparison with culture and serology using samples from patients with suspected whooping cough from a highly immunized population. J Infect Dis. 1996;174(1):89-96.

21. Klein NP, Bartlett J, Rowhani-Rahbar A, Fireman B, Baxter R. Waning protection after fifth dose of acellular pertussis vaccine in children. N Engl J Med. 2012; 367(11):1012-9.

22. Misegades LK, Winter K, Harriman K, Talarico J, Messonnier NE, Clark TA, et al. Association of childhood pertussis with receipt of 5 doses of pertussis vaccine by time since last vaccine dose, California, 2010. JAMA. 2012; 308(20):2126-32.

23. Tartof SY, Lewis M, Kenyon C, White K, Osborn A, Liko J et al. Waning immunity to pertussis following 5 doses of DTaP. Pediatrics. 2013; 131(4):e1047-52.

24. Witt MA, Katz PH, Witt DJ. Unexpectedly limited durability of immunity following acellular pertussis vaccination in preadolescents in a North American outbreak. Clin Infect Dis. 2012; 54(12):1730-5.

25. Rohani $P$, Zhong $X$, King $A A$. Contact network structure explains the changing epidemiology of pertussis. Science. 2010; 330(6006):982-5.

\section{Acknowledgements}

The authors wish to thank individuals and organizations across health authorities for their contribution to these surveillance data. In particular, we would like to acknowledge the following individuals: Dr. Mark Bigham and Stephanie Konrad (Fraser Health Authority); Sara Forsting (Vancouver Coastal Health Authority); Dr. Dee Hoyano (Vancouver Island Health Authority); and Yin Chang (Public Health Microbiology Reference Laboratory, Provincial Health Services Authority).

\section{Conflict of interest}

No conflicts of interests to declare.

\section{Funding}

None 